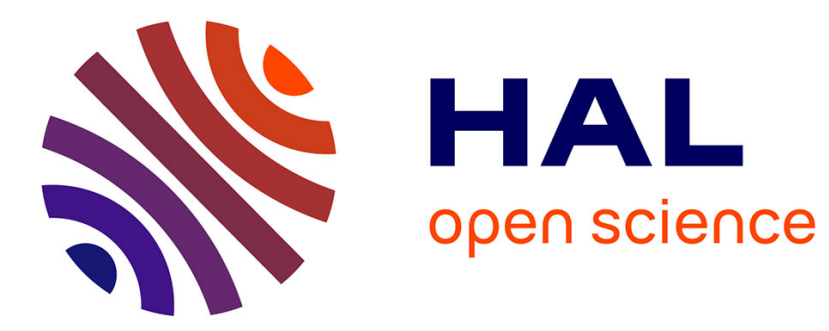

\title{
Sudden cardiac death and Turbulence
}

Guillaume Attuel, Oriol Pont, Binbin Xu, Hussein M. Yahia

\section{To cite this version:}

Guillaume Attuel, Oriol Pont, Binbin Xu, Hussein M. Yahia. Sudden cardiac death and Turbulence. The 8th International Conference (CHAOS2015) on Chaotic Modeling, Simulation and Applications, May 2015, Paris, France. hal-01207139

\section{HAL Id: hal-01207139 \\ https://hal.inria.fr/hal-01207139}

Submitted on 1 Oct 2015

HAL is a multi-disciplinary open access archive for the deposit and dissemination of scientific research documents, whether they are published or not. The documents may come from teaching and research institutions in France or abroad, or from public or private research centers.
L'archive ouverte pluridisciplinaire HAL, est destinée au dépôt et à la diffusion de documents scientifiques de niveau recherche, publiés ou non, émanant des établissements d'enseignement et de recherche français ou étrangers, des laboratoires publics ou privés. 


\title{
Sudden cardiac death and Turbulence
}

\author{
Guillaume Attuel ${ }^{1}$, Oriol Pont ${ }^{1}$, Binbin $\mathrm{Xu}^{1}$, and Hussein Yahia ${ }^{1}$ \\ GeoStat, "Geometry and Statistics in acquisition data", Centre de recherche INRIA \\ Bordeaux Sud-Ouest, 200 rue de la Vieille Tour, 33405 Talence Cedex, France \\ (E-mail: guillaume.attuel@inria.fr)
}

\begin{abstract}
Data acquired from the electrical activity of human hearts during episodes of atrial fibrillation, a disordered arrhythmia that is a major cause of stroke, reveals intriguing features for an excitable media: highly skew symmetric probability distributions with heavy tails, long range correlations, and broad singularity spectra. Interestingly, the relevant exponents extracted from these empirical laws are stable over several minutes but not universal. Their stable values are distributed among patients and areas of the heart. The question of central clinical purpose is whether they might characterise locally the myocardium contingent pathology. To achieve clarification of these peculiar facts, we were led to devise a phenomenological model that departs from the conventional approach to fibrillation. Instead of a defect mediated spiral wave "turbulence" induced by front collisions, fibrillation is pictured here as a highly intermittent modulation of cardiac pulse trains. It is based on the physiology of inter-cellular ionic exchanges, which is associated with the natural degree of freedom of the inter-pulse duration. We infer an experimentally unknown slow dynamics of inter-cellular coupling, that may induce an inter-pulse effective coupling. This interaction creates a modulation that may lead to intermittency in various ways. The exchange of charges occurs at small scales in the model. They are passively advected at each interstitial junction on fast time scales and on average collectively driving the larger scales. In fact, a dimensionless number characterising the dynamics is an analogue of the Rayleigh number. Away from a rapidly beating source, random back scattering and front splitting make pulses follow random hierarchical "percolating" paths in $1 \mathrm{D}$. We discuss very briefly the topological origin of these dynamics. In the light of this model, we don't omit to mention some important physiological aspects of the pathology that are still not well understood and more possibilities for the case which comes to grip with sudden cardiac death.
\end{abstract}

Keywords: Heart dynamics, Reaction-Diffusion, Driven systems, Self organized criticality, Multiplicative noise, Random paths, Topological excitations, Singularities.

\section{Introduction}

Sudden cardiac death accounts for about $10 \%$ of all natural deaths in developed countries and for about $50 \%$ of the mortality from cardiovascular diseases. Meanwhile, it is not really a pathology: statistical surveys have found risk factors but which fail to be individually predictive [1]. In our present understanding, it can be considered more as an accident. One cause of sudden cardiac death is fibrillation of the ventricles. Experimental studies are difficult for obvious reasons. The milder case of atrial fibrillation (AF), which is however one 
major cause of stroke in Europe and North America, can be monitored and studied more easily. In this paper, we give the general scheme of an analysis of $\mathrm{AF}$ that we are developing. We will shortly discuss at the end some possible lessons we may learn from it for sudden cardiac death.

The heart muscle is an excitable tissue, long believed to be a syncytium of myocardial cells. Models of excitability for the heart are reaction-diffusion systems that describe the propagation of electrical pulses, called action potentials. They result from ionic exchange cycles between the cytoplasm of excitable cells and their extra-cellular medium. A typical example is an action potential propagating through a nerve axon [2] or throughout the myocardium [3] [4] [5] [6]. In the right atrium of a human heart, the sinus node (a pacemaker) ensures a periodic stimulation of the tissue, from where pulses propagate regularly in normal sinus rhythm. In its abnormal states, called arrhythmias, the myocardium is overwhelmed by rapid and irregular patterns of activation. In part for the reasons we are exposing in the text, we consider AF the most irregular arrhythmia, as illustrated in fig.(1).
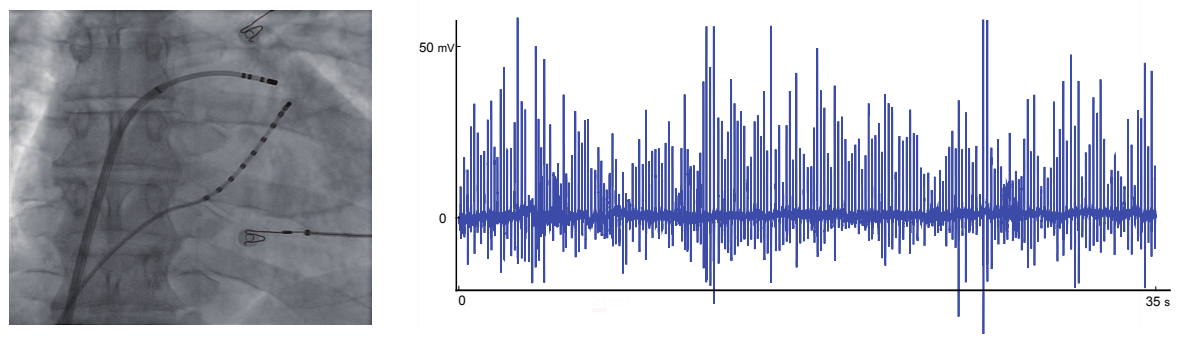

Fig. 1. $35 \mathrm{sec}$ of paroxysmal $\mathrm{AF}$ at a sampling rate of $1 \mathrm{kHz}$ are shown recorded from a bipolar electrode, with some peaks reaching saturation level of the recording device. Electrodes are about $2 \mathrm{~mm}$ apart. A radiography of the left atrium is shown, where the bipolar electrode is located near the superior left pulmonary vein, held by a medical practitioner, whereas the other catheter comprising 10 electrodes runs along the coronary sinus.

Chaos has been observed in cultures of automatic cardiac cells, through their coupling to periodic stimulation, and also in the heart via the occurrence of parasystoles. This can be traced to phase locking and chaos of relaxation oscillators with periodic forcing, and could be well modelled by return maps [7] [8]. Also, period doubling, called alternans in this context, arises when the adaptation of the action potential duration to an imposed rate becomes unstable [9]. For this reason, alternans are thought in one hypothesis as a route to ventricular fibrillation [10]. Moreover, fibrillation in the atria were in some instances clinically found to follow an inadaptation of the action potential duration [11]. However, an excitable limit cycle is very robust when it comes down to chaos, because its saddle fixed point does not give way to a homoclinic tangle. This can be understood by considering the stable fixed point as an absorbing state, or a phase resetting state. Therefore, even under periodic 
forcing, no return map can be drawn. In other words, the regularity of the triggering sources is transferred to the limit cycle.

In contrast, as propagation becomes further involved in the arrhythmia, the usual theoretical interpretation is based on a kind of defect mediated turbulence, specific to excitable oscillators. Basically, spiral waves are often found more stable than striped or other patterns in excitable media. This stems from the important fact that an excitable pulse with a free end meets a region in a refractory state and starts wandering about it [12] [13] [14]. It is also more fundamentally related to the topological charge of the spiral core [15] and to the chiral symmetry breaking that an excitable pulse carries with itself (a Bloch wall) [16] [17] [18]. Low dimensional aperiodicity has again been observed before the onset of fibrillation for meandering spiral cores [19]. To account for spatio-temporal chaos a mechanism of front collisions is put forward. If in oscillatory media spirals may break up due to a modulational instability of the emitted pulse train from its meandering core, in excitable media the breakup seems to amount to direct fore front and back front collisions within the pulse train [20] [21] [22]. Notice that when a small diffusivity of the inhibitor is added, a curvature instability may lead to front splitting and spiral turbulence near the Ising-Bloch transition [23].

As successful as low dimensional chaos, and spatio-temporal chaos, in excitable media may look in describing these arrhythmias, we show here why this paradigm is quite inadequate to tackle an essential property of the recorded data, which hasn't been noticed so far.

A crucial aspect about the pathology is the intermittency of bursty occurrences of the arrhythmia. Intermittent alternation of sinus rhythm with fibrillation is what defines paroxysmal AF. This intermittency can be modulated by the external drive of the autonomic nervous system, as shown by P. Coumel and co-workers, see for instance [24]. Moreover, on the myocardium surface, within each episode of fibrillation, the recorded signal is found to be more or less regular: the so called "fragmentation" in clinical lingo. In fact, as we demonstrate in section 2 , the locally recorded electrical potential exhibits many traits in common with hydrodynamic intermittency. Another poorly understood fact is a reversible process of deterioration of the myocardium during AF called remodelling [25]. It appears that the longer the heart remains in a fibrillatory state, the poorer its conduction properties become, and the more stable this abnormal state gets. We believe that these features are rooted in some underlying chemical modulation of the electrical synapses between the cells. Our model demonstrates this possibility.

We may look for the nucleation of metastable chaotic domains to explain such observations. This has led us to find out a physiological path to the mechanism described by Y. Pomeau [26]. As illustrated in [27], if a limit cycle is able to reach its homoclinic connection, then bistability with another fixed point, or cycle, may generate spatio-temporal intermittency. The difficulty is that an excitable limit cycle is over-damped and very robust. No such saddle fixed point connecting with another basin of attraction normally exists. Even worse would be to try to find a crisis bifurcation to some strange attractor. Never mind, if the tissue is in fact capable of generating another limit cycle, then the route 
devised by Y. Pomeau might be reached. For instance a modulation of pulse trains would work. This can be indeed the case as we show. This originates in the electrical coupling between the cells, that under some circumstances which we describe, may grow collectively, versus diffusively. One can thus rephrase the previous findings for oscillatory media in terms of these collective modes, and find a variety of interesting scenarios. For simplicity, we adopt a point of view à la Ginzgurg Landau.

We formulate therefore in section 3 a derivation from first principles of the yet unknown dynamics of ionic currents at the gap junctions. This dynamical coupling between cells is considered as a synaptic plasticity. The point is to question the importance of intrinsic fluctuations and disorder. We make sure that observing the Ginzburg region of criticality in excitable reaction-diffusion systems is classically impossible. Then, we will find out that cardiac pulses can be pinned where cycles of neighbouring cells become out of phase with one another, because of interstitial plasticity. This unfortunate plasticity may slowly contaminate the whole tissue, which is what we will relate to electrical remodelling.

In section 4 , we very briefly sketch an interpretation. Due to the intrinsic noise, the critical Ginzburg domain extends widely, leading to self organised criticality (SOC). This maps to multiplicative noise, describing the singularities in the signal.

We will show throughout the text the high level of agreement between the patients data and the model data.

\section{Time series, fluctuations and limitations of excitable models}

Surface electrical potentials recorded as time series during AF are called electrograms (egm), such as the one shown in fig.(1). Normal frequency $f$, in beats per minute, is about $f \approx 60 \mathrm{bpm}$, whereas during AF, it is typically in the range of $200 \mathrm{bpm} \lesssim f \lesssim 600 \mathrm{bpm}$. At first sight, egms during AF contrast to normal as they seem to fluctuate randomly. Their amplitude also looks locally abnormally oscillatory, fig.(2). The auto-correlation function starts decreasing rapidly, exponentially fast during the first $60 \mathrm{~ms}$ or so, and goes on oscillating with a slow decrease of the envelop, asymptotically as a power law, see fig.(2), where an indicative solid line $\sim t^{-1}$ is drawn. Peaks appear naturally as multiples of the average periodicity of the arrhythmia at about $300 \mathrm{bpm}$ there. The identification of the shorter time scale is expected and indicative of local incoherent oscillations, but the algebraic correlation law of the envelop is more suggestive of some collective phenomena with quasi long-range order. Thus, we are led to look for some collective modulation of the pulses.

We observe that fluctuations are large and their probability density distributions collapse as is seen in fig.(3). The high skewness and heavy tails are a hint of underlying mechanisms. They can be cast into the form

$$
P\left(A, A_{c}\right)=A^{-\tau} G\left(\frac{A}{A_{c}}\right)
$$



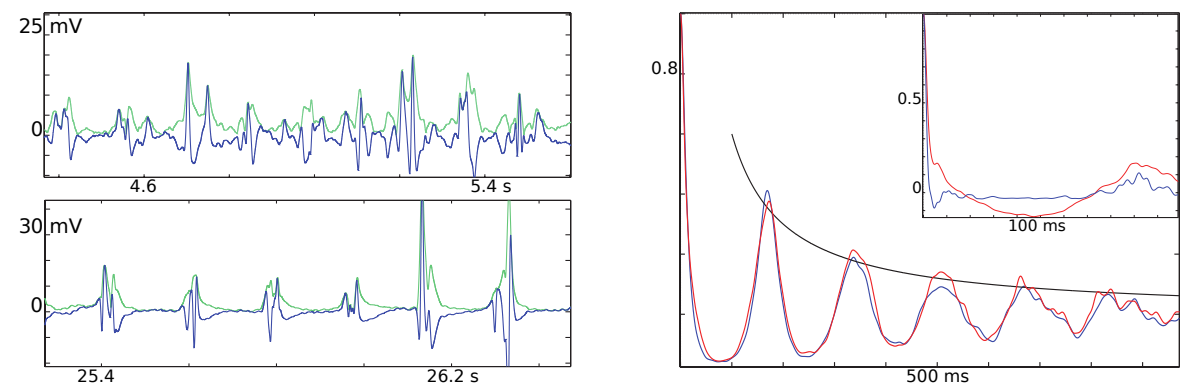

Fig. 2. Left: Two instances of one egm amplitude and its envelop at two different moments in time, where a Hilbert transform was used. Right: Two auto-correlation functions of egm envelops, and of amplitudes in the inset, for two different locations on the heart.

where $A$ is the egm varying amplitude in $m V, A_{c}$ is a cut-off, $\tau$ is a scaling exponent, and a scaling function $G$ decreasing rapidly towards zero. Very briefly summarized, various values of $\tau$ have been found. They range roughly between $1.2<\tau<3$ among patients, and regions of the atria. To our knowledge, simi-
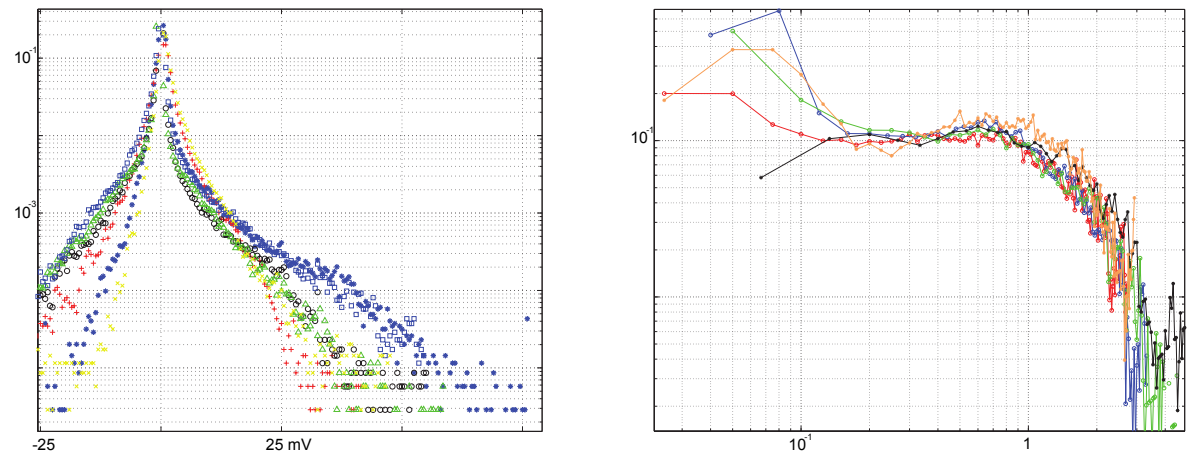

Fig. 3. Normalised probability density distributions of egm amplitudes in semilog scale, from all over the atrium of one patient (left). Shown in loglog scale is the empirical collapse, for positive values and various exponents $\tau$, on a scaling function $G$ of eq.(1) (right). $A_{c} \approx 30-50 \mathrm{mV}$.

lar fluctuations were not found in excitable systems, but are rather ubiquitous in complex systems. To name a few instances, they are found in random field Ising models [28], with the Barkhausen noise as a magnetic field is applied to a dirty ferromagnet [29], for magnetic penetration in hard superconductors [30] [31] [32], during the firing activity of some neural networks [33][34][35][36], or in the intermittent regimes of strong turbulence [37] [38]. 
Turning to continuous excitable media, they are modelled by reactiondiffusion systems

$$
\left\{\begin{array}{l}
\frac{\partial}{\partial_{t}} U_{m}=\mathcal{R}\left(U_{m}, J_{m}\right)+D \Delta\left(U_{m}\right) \\
\frac{\partial}{\partial_{t}} J_{m}=\mathcal{G}\left(U_{m}, J_{m}\right)
\end{array} .\right.
$$

Here, $U_{m}$ is associated with the membrane potential of a cell, and $J_{m}$ is a vector representing (non diffusing) inhibitors, associated with the many ionic currents going through channels across the otherwise impermeable membrane of a cardiac cell. In the limit of interest, time scales are well separated, that is $U_{m}$ is a fast variable, while $J_{m}$ is a slow variable. One usually denotes by $\mu^{-1} \approx 1 \mathrm{~ms}$, the typical fast time scale, which corresponds to the time for the insulating membrane, of thickness $\Lambda \approx 10 \mathrm{~nm}$, to depolarise. This is possible at such short time scales because Nernst-Planck thermal equilibrium is reached indeed thanks to facilitated diffusion and active pumping of ions [39]. The slow time scale, associated with repolarisation, is typically of the order of $100 \mathrm{~ms}$ or greater.

Now, to be endowed with the property of excitability, the system's null clines, $\mathcal{R}=0$ and $\mathcal{G}=0$, basically intersect in a way as to produce locally a kind of saddle-node configuration. Nonlinearities and dissipation (or periodic order parameters) give rise to a limit cycle once an orbit is generated away from the saddle fixed point. It is insightful to draw a straightforward analogy with a Van der Waals diagram. Roughly speaking, the analogy goes as: a cycle "nucleates" each time the "supercooled spinodal branch" is reached by a finite perturbation. The width of the nucleation region corresponds to the degree of excitability. A Ginzburg-Landau description of eq.(2) reads as $\frac{\partial}{\partial t} U_{m}=-\frac{\delta}{\delta U_{m}} \mathcal{F}_{0}$, with a free energy of the form

$$
\mathcal{F}_{0}=\frac{1}{2} \int d x^{d}\left\{-\mu U_{m}^{2}+\frac{\beta}{2} U_{m}^{4}+D\left(\nabla U_{m}\right)^{2}-I U_{m}\right\}
$$

where all parameters are positive, $I=J_{0}-J_{m}$ is a source term, and $J_{0}$ is an external input of current. Dimension $d=2$ is appropriate for the atria, since the atrial myocardium is very thin, typically of order $2 \mathrm{~mm}$ thick, as it does not contribute much to the pump function of the heart, while $d=3$ is more adequate for the ventricles. In fact dimension $d=1$ is quite appropriate also for the description of fast conducting fibres in both chambers.

Since the free energy has two local minima, depending on boundary conditions, domain walls typically form. In source free conditions, their height is $U_{0}=2 \sqrt{\frac{\mu}{\beta}} \approx 100 m V$ and their thickness is the Ginzburg-Landau correlation length $l_{c} \propto \sqrt{\frac{D}{\mu}} \approx 1 \mathrm{~mm}$. They propagate at constant velocity by diffusion, with a velocity $c \propto \sqrt{D \mu} \approx 1 \mathrm{~ms}^{-1}$. The role of the recovery current $J_{m}$ is to break the symmetry between the two minima of the energy manifold, by favouring the return to one of them, corresponding to the rest potential. In its most basic version, we have the Fitzhugh-Nagumo model (FhN), with $\mathcal{G}\left(U_{m}, J_{m}\right)=\gamma U_{m}-\sigma J_{m}-\eta$, where $\sigma$ controls the repolarisation time scale, and $\eta$ is a leaking current. It defines a cell cycle. 
For the sake of simplicity here, let us take $\eta=0$, the condition of excitability becomes $\mu>\frac{\gamma}{\sigma}$. FhN, in the sense of a generalised Van der Pol set of equations, spontaneously evolves into chaotic states, for instance under periodic forcing [40] or with more complicated reactions $\mathcal{G}$ [41]. Configuration averaging leads to an effective reparametrization $\mu \rightarrow \mu-\frac{\gamma}{\sigma}$, since on average one expects fast modes to be slaved to slow modes, $\left\langle J_{m}\right\rangle=\frac{\gamma}{\sigma} U_{m}$. The mean field susceptibility may then increase to very high values $\chi \sim\left(\frac{\gamma}{\sigma}-\mu\right)^{-1} \rightarrow \infty$, explaining the large fluctuations and long range correlations observed.

The argument above fails firstly because it only tells us locally that cycles will be triggered almost with no threshold. Secondly, on a global scale, the narrowness of the critical Ginzburg region in parameter space prevents any wild collective effect to become observable [42]. Taking the order of magnitude of the diffusive length of about $l_{c} \approx 1 \mathrm{~mm}$ and bringing it next to the microscopic cut-off length, the maximum between the gap junction wall thickness $\Lambda \approx$ $100 \mathrm{~nm}$ and the Debye length, here about $\lambda_{D} \approx 10 \mathrm{~nm}$, one obtains a very narrow width of the parameter range, entirely unobservable in practice $\delta \chi^{-1} \sim$ $\Lambda^{2} l_{c}^{-2} \lesssim 10^{-10}$.

This rules out near equilibrium critical fluctuations in ordinary excitable media. We have realized however that mean field arguments break down when the ionic exchange current at the gap junction alters the effective potential energy of the cell, in such a way as to restore a continuous symmetry, and approach an effective critical region.

\section{Incorporation of cell to cell dynamical coupling}

The works in [43] [44] [45] show the crucial role played by the gap junctions, since they are supposed to guarantee good coupling between the cells. However, in excitable models it is not clear how bad conduction can be modelled. According to near equilibrium thermodynamics, the exchange current may simply be written down as $J_{e}=-g_{s} \nabla U_{m}$, where $g_{s}$ is a stationary conductance. The point is to demonstrate that the perturbation of the opening and closing of the gap junction channels induce some time lag in the activation of the cell.

Typical relaxation times of gap junctions are much larger than those of membrane polarisation, but compare well with membrane repolarisation time scales. On average they are of order $\gtrsim 100 \mathrm{~ms}$ [46]. There is thus no alternative but to consider the full kinetics of the gap junctions, at such high frequencies as found in arrhythmias. This is a crucial aspect missing in common models for arrhythmias. Applying common wisdom on membrane physico-chemistry to the gap junctions, replacing $U_{m}$ with $\nabla U_{m}$, yields a kinetic relaxation equation of the form [2]

$$
\frac{\partial}{\partial t} g=a\left(\nabla U_{m}\right)\left(g_{0}-g\right)-b\left(\nabla U_{m}\right) g,
$$

where the functions $a\left(\nabla U_{m}\right)$ and $b\left(\nabla U_{m}\right)$ are the respective average rates of opening and closing of the gap junction channels. The constant $g_{0}$ is a typical maximum resting value, essentially $g_{s}=g_{0} \frac{a}{a+b}$. The gradient is the one felt at the gap junctions. So we turn to the question of how the electrical force $-\nabla U_{m}$ 
can be strong enough as to perturb $a$ and $b$ in order to make the current deviate from electro-diffusion. Since a pulse front of typical width $l_{c}$ encompasses many cells, the voltage difference $\sim \Lambda \nabla U_{m}$ at a gap junction cannot be as strong as the one felt across membranes. Nevertheless, gap junction channels have a very distinctive role in inter-cellular communication. They are open at rest state, and very long molecules permeate through. They are therefore inclined to a modulation of their permeability, depending on the concentration levels of some messenger molecules [47]. We explore here this possibility, letting some ions act on the gap junction properties. This will simply arise from their naturally slow linear response to the presence of high ionic concentration.

In that respect, there exists a point of view that allows us to characterise the ionic flow by a dimensionless number. For certain values of this dimensionless number, an instability will occur for the most unstable mode [48], which eventually will develop dissipative structures [49][50]. Upon forcing the system to higher values, secondary instabilities may destabilize the primary structures, leading to a broad spectrum of modes [51].

To construct our model, we basically use charge conservation and a kinetic equation for the gap junction channel average opening under proper thermodynamic forcing. As is depicted in fig.(4), we consider an excess charge sitting at the gap junction, and its effect on the equilibrium dynamics. The force

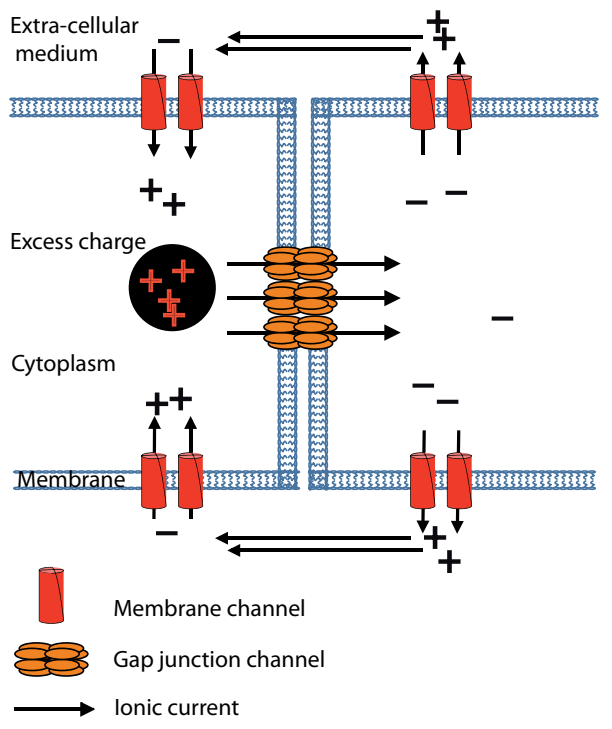

Fig. 4. Excess of positive charge (black) sitting next to the gap junction channels. Arrows indicate inter-cellular flows, which are generally supposed to be diffusive.

is simply the electro-chemical gradient. In $1 \mathrm{D}$, as the sketch suggests, take a finite volume element $\mathcal{V}$ spanning the gap junction, incorporating the excess charge and extending to the membranes. Noting $\rho$ the excess density, charge 
conservation inside this volume reads $\frac{\partial}{\partial t} \rho=-\tilde{g} \nabla U_{m}$, where the gradient is understood as a finite difference over the closed surface. We used a perturbed conductance $\tilde{g}$, which is the important assumption in our model. It states that excess charge density variations overrate the stochastic averaging of the opening and closing of the channels, that would otherwise set the conductance to its equilibrium value. Therefore, we need to consider eq.(4), which we will linearise as $a\left(\boldsymbol{\nabla} U_{m}\right)=\alpha \rho+a_{0}$, with $\alpha$ a control parameter, and $b=b_{0}$ for the sake of simplicity of the demonstration. This linearisation simply stipulates that the excess charge amounts to $\mathcal{V} \rho \approx C \nabla U_{m}$, with a gap junction effective capacitance $C$. Note that the extra cellular medium is supposed to rest at a constant potential reference.

The combined equations basically say that excess charges are swept along the small scale gradients (excess charges will tend to average out over large volume elements comprising many cells), while variations of the conductance remain local. Denoting $\nu=a_{0}+b_{0}$, dropping the tilde for clarity, we get the following system of equations

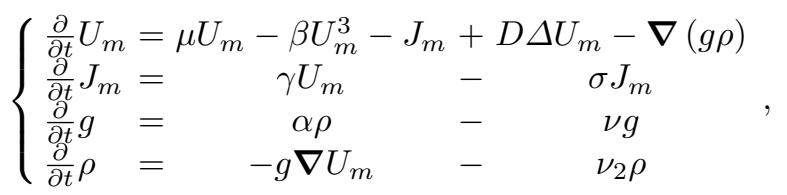

where we have let the capacitance and volume $C \equiv 1, \mathcal{V} \equiv 1$ without loss of generality. The locally perturbed current is $g \rho$ by construction. The evaporation rate $\nu_{2}$ is a local simplification of charge diffusion, for a fixed length scale, and is meant to be small. This set is not parity invariant, and by construction one needs to take an opposite $\alpha$ to change directions of front propagation from the location of a source, since the potential gradient will reverse sign.

As we described above, some important perturbations of the dynamics may emerge at slow time scales. Indeed, this simple model is in spirit quite comparable to a kind of Rayleigh instability, where $\alpha$ plays the role of the gravitational pull. Because the interface is fixed at the gap junction, no convective term is present. More precisely, when only two cells are coupled with one free boundary, notice indeed how the first, third and fourth equations have the same structure as the Lorenz system of ODE (where the opposite limit $\mu \rightarrow 0$ holds though). The analogue Rayleigh dimensionless number is here $\mathcal{R}_{a} \equiv \frac{\alpha \rho}{L \nu \nu_{2}}$, which controls the effect of thermodynamic forcing over dissipation, where $L$ is an equilibrium length associated with the slow time scales. In fact, we force the system at one end with an automatic cell (a very rapid abnormal pacemaker), or similarly with an abnormal current leak $J_{0}$. Now, since on average we will have $D\left\langle\frac{\rho}{L}\right\rangle \approx J_{0}$, it is possible to rewrite

$$
\mathcal{R}_{a} \equiv \frac{\alpha J_{0}}{D \nu \nu_{2}}
$$

So we expect a transition point towards chaos around $\mathcal{R}_{a} \sim 1$, for very small arrays, of two to a very few cells, and to turbulence in longer arrays. This transition to high dimensional chaos is illustrated in fig.(5). 

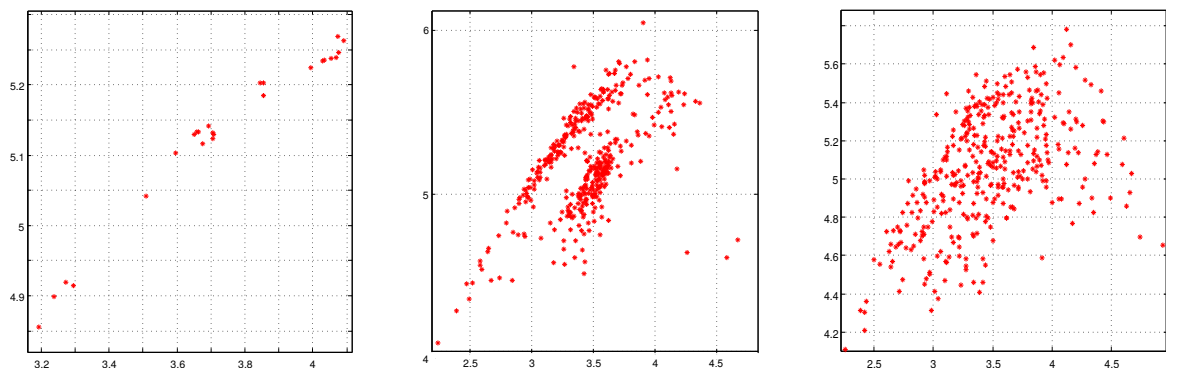

Fig. 5. Poincaré section plots $(\rho, g)$, from maxima of $U_{m}$, for 2,3 and 4 cells coupled linearly, in the special case $\mu=0$ and $\sigma \rightarrow \infty$. The section on the left is from the famous Lorenz attractor. One notes the spreading of points revealing the increase of the attractor dimension.

It is easy to quickly check the validity of this argument numerically. Starting with parameters for which we observe regularity of beats and rhythm, decreasing $D, \nu_{2}$ or $\nu$, and raising $\alpha$ makes it possible to reach a domain of turbulent dynamics of $\rho$ and $g$, that strongly affect $U_{m}$ and $J_{m}$, see fig.(6). Here, we provide an illustration of the turbulent domain with the same numer-
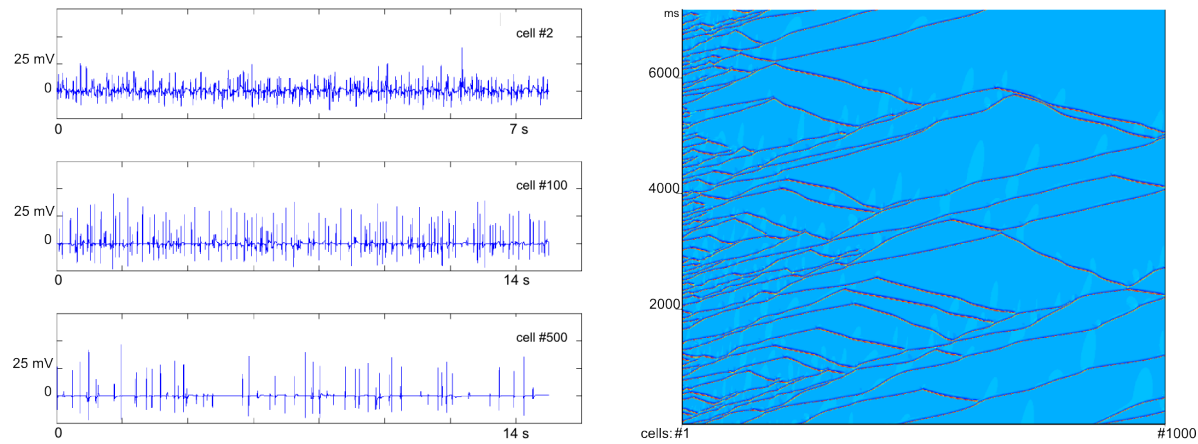

Fig. 6. Some traces of the gap current divergence, in the model in $1 \mathrm{D}$, from near a source of abnormal automaticity, cell \#0, to further away. Spatio-temporal map of action potentials showing many back-scattering and some front splitting in a hierarchical structure of propagation, since the ones that escape collisions rarefy. $\mu=1$, $\beta=1, \gamma=0.008, \sigma=0.02, \alpha=0.01, \nu=0.01, \nu_{2}=0.0001$

ical values of the parameters as in fig.(6). We do find similar properties for the numerical data as for the experimental data. It seems indeed that long range auto-correlations decrease as $\sim t^{-1}$ power laws. As shown in fig.(7), probability density distributions of the current divergence scale in the same way. Despite that the effect of the system size seems negligible at first glance on the onset of turbulence, we find non universal exponents, which appear to mark the distance from the source. Just as strikingly, the broad singularity spectra, with a 

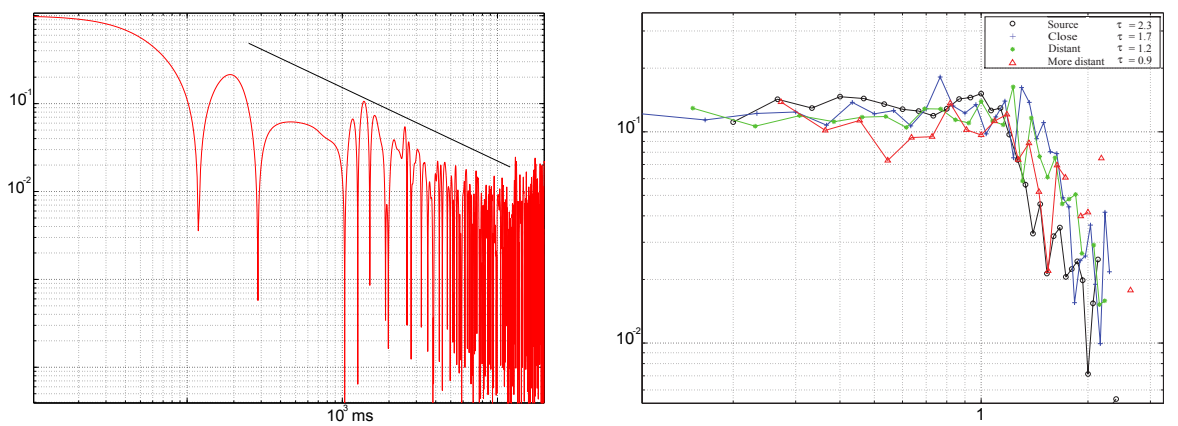

Fig. 7. Loglog plot of the auto-correlation of cell \#5, with an indicative $t^{-1}$ plot (left). Empirical collapse function $G$ for the model (right), with non universal exponents, decreasing with distance from the source for arbritrary cells, shown \#2, \#20, \#80, and \#400.

substantial contribution of negative exponents, can be superposed completely, see fig.(8). This tends to demonstrate the presence of an identical random cascade process underlying the dynamics.
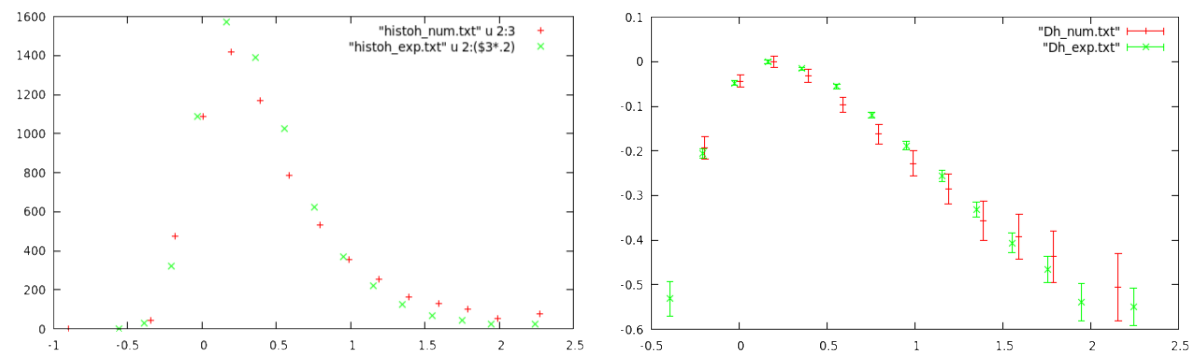

Fig. 8. Strikingly good superposition of the broad histograms (left) and fractal dimension spectra (right) of the sets of singularity exponents $h$ defined locally, for small $\tau$ of a few $m s$, as $\left\langle\int^{\tau} d t \frac{\partial}{\partial t} \nabla g \rho\right\rangle \sim \tau^{h}$. They were obtained from the same experimental data as in fig.(2) and the $1 \mathrm{D}$ numerical data as in the previous figures for an average over 10 cells taken at random spanning the first 100 cells.

The transient time that the turbulent state takes to pervade the system could be related to the electrical remodelling. We observe a typical time scale that reads like $T \sim L^{z}$, with $z \approx 1$. For typical length of human atrial fibres, it happens to fall in the physiologically recorded range of about a few minutes [52]. Finally, although system size does not affect the onset of turbulence as expected, the distance from the source affects the scaling exponents we have found, see fig.(7). This basically marks the hierarchical propagation pattern. In practice, this could be good news for a quantitative method of finding abnormal sources of activity in the heart, a highly valued goal pursued by medical practitioners and physiologists. 
This phenomenology holds in two dimensions with isotropic coupling as well. Note also that the propagation of perturbing charges is like some effective diffusion of the inhibitor. Therefore, considering the anatomical organisation of the myocardium in fibre bundles and the anisotropy of conducting properties, one expects fronts to split along their direction.

By chance, in three dimensions various topological arguments convey the idea that the ventricles are better equipped to resist such onset of very irregular patterns. Thus far, one may have in mind natural selection to understand the Aschoff-Tawara node, which function is somehow to low-pass filter the activity of the atria, before relaying it to the ventricles.

\section{Discussion and conclusion}

The large oscillations of pulses and the intermittency are quite intriguing at first, since $\mu$ is the dominant parameter, which guarantees the stability of $U_{0}$ against any spontaneous fluctuation. In fact, a phase approximation of the dynamics is indeed relevant in this sector. Then, what is seen might signal the restoration of a continuous symmetry for the dynamics of the phase, that finds itself effectively at criticality.

Firstly, upon appropriate rescaling, define a complex scalar $\Psi=U_{m}+i J_{m}=$ $A e^{i \theta}$. The phase $\theta(\mathbf{x}, t)$ is a distribution of ticks recording the passage and shape of pulses. Since $\mu$ defines the rapid time scale, it is natural to consider a fixed amplitude of $\Psi$. Let us model the perturbation caused by the ionic gap currents as some local time delay $\varphi$ for the onset of depolarisation. The equation for the phase then reads

$$
\partial_{t} \theta=D \Delta \theta-H \sin (\theta+\varphi(\mathbf{x}))+F,
$$

where $H$ and $F$ define characteristic scales that can be made to match that from $\mathcal{R}$ and $\mathcal{G}$, such as the domain wall thickness $l_{c} \sim \sqrt{H^{-1}}$. Taking a random distribution of phase in the range $\varphi(\mathbf{x}) \in[0,2 \pi]$, random pinning is facilitated. This governs the behaviour of charged density waves in impure magnetic materials [53] [54] [55] [56]. Naively, an effective critical state could be reached from the average of random phases $\mu_{e f f} \sim\langle H \cos (\varphi)\rangle$, though the model equation does not reduce to critical dynamics, model A in [57]. Basically, one can find in the literature the anomalous scaling of the velocity jumps of the density waves, that reads like $\delta v \propto F^{\xi}$ with $\xi \neq 1$, near the forcing threshold of the depinning transition (insulating to conducting). SOC is typically found in those systems [58]. Counting consecutive phase slips, one finds a distribution of avalanches that typically scales with system size, a cut-off measuring a distance to a critical point, in a form like eq.(1), where the exponent $\tau$ is related to $\xi$ [59] [28].

Hence, one notes that avalanches of phase slips, within a surrounding closed contour, must be related to large amplitude variations of the bulk average. Heuristically, the argument is quite suggestive of multi-scaling. From the slowly varying random aspect of the noise term emerges a random cascade. It is tempting to model this dynamical effect by a mean field multiplicative 
noise $\mu \mapsto \tilde{\mu}\left(J_{0}, \boldsymbol{x}, t\right)$ acting on top of diffusion, leading to large deviations as captured by the observed singularity spectra [38], and percolating paths [60].

In conclusion, we have presented data, from humans with a very irregular arrhythmia, that seem to exhibit patterns of hydrodynamic intermittency. We showed that such fluctuations could not emerge from purely excitable dynamics, and found out a good alternative candidate, namely intrinsic modulations. We devised a model of ionic flows through the gap junction channels of a cardiac tissue, that effectively modulate otherwise independent pulses. The observed abnormal patterns finely match the ones from the model, when the flow is intermittent. It is the first to manifest a transient related to the degradation of pulse propagation, called electrical remodelling, and to suggest a relationship between local exponents in the signal with the distance to an abnormal source.

In that respect, we would like to believe that our model may further illustrate Y. Pomeau's conjecture, relating hydrodynamic intermittency with some directed percolation of metastable orbits.

At any rate, these results are clear evidence of the role of the dynamical coupling of the network of cells, which do not form a true syncytium.

\section{References}

1. D. P. Zipes and H. J. J. Wellens. Circulation, 98:2334, 1998.

2. A. L. Hodgkin and A. F. Huxley. J. Physiology, 117(4):500, 1952.

3. B. van der Pol and J. van der Mark. Phil. mag. series 7, 38(6), 1928.

4. D. Noble. J. Physiology, 160:317, 1962.

5. R. Fitzhugh. Mathematical models of excitation and propagation in nerve. H.P. Schwan (Ed.), Biological Engineering, McGraw-Hill, New York, 1962.

6. J. Nagumo, S. Arimoto, and S. Yoshizawa. Proc. IRE, 50:2061, 1962.

7. M. R. Guevarra and L. Glass. J. Math. Biology, 14:1, 1982.

8. L. Glass, M. R. Guevarra, A. Shrier, and R. Perez. Physica 7D, page 89, 1983.

9. M. R. Guevarra and L. Glass. IEEE Computers in Cardiology, page 167, 1984.

10. A. Karma. Chaos, 4:461, 1994.

11. P. Attuel et al. Int. J. Cardiol., 2:179, 1982.

12. M. A. Allessie, F. I. M. Bonke, and F. J. G. Schopman. Circ Res., 41(1):9, 1977.

13. E. Meron and P. Pelcé. PRL, 60(18):1880, 1988.

14. A. Hagberg and E. Meron. Phys. Rev. Lett., 72(15):2494, 1994.

15. I. S. Aranson and L. Kramer. Rev. mod. Phys., 74(1):99, 2002.

16. J. Lajzerowicz and J. J. Niez. Journal de Physique Lettres, 40(7):165, 1979.

17. P. Coullet, J. Lega, B. Houchmanzadeh, and J. Lajzerowicz. Phys. Rev. Lett., 65(11):1352, 1990.

18. T. Frisch, S. Rica, P. Coullet, and J. M. Gilli. Phys. Rev. Lett., 72(10):1471, 1994.

19. A. Garfinkel et al. J. clinical investigation, 99(2):305, 1997.

20. A. Karma. Phys. Rev. Lett., 71(7):1103, 1993.

21. A. T. Winfree. Science, 266(5187):1003, 1994.

22. F. H. Fenton, E. M. Cherry, H. M. Hastings, and S. J. Evans. Chaos, 12(3):852, 1993.

23. A. Hagberg and E. Meron. Chaos, 4(3):477, 1994.

24. P. Attuel, P. Coumel, and M.J. Janse. The atrium in health and disease. Mount Kisco, NY: Futura Publishing Co., 1st edition, 1989.

25. M. C. Wijffels, C. J. Kirchhof, R. M. Dorland, and M. Allessie. Circulation, 92:1954, 1995. 
26. Y. Pomeau. Physica 23D, page 3, 1986.

27. M. Argentina and P. Coullet. Phys. Rev. E, 56(3):R2359, 1997.

28. K. Dahmen and J. P. Sethna. Phys. Rev. B, 53(22):14872, 1996.

29. J. C. McClure Jr. and K. Schroder. C R C Critical Reviews in Solid State Sciences, 6(1):45, 1976.

30. J. S. Urbach, R. C. Madison, and J. T. Market. Phys. Rev. Lett., 75(2):276, 1995.

31. C. J. Olson, C. Reichhardt, and Franco Nori. Phys. Rev. B, 56(10):6175, 1997.

32. E. Altshuler et al. Phys. Rev. B, 70:140505, 2004.

33. J. M. Beggs and D. Plenz. J. Neurosci., 23(35):11167, 2003.

34. C.-W. Shin and S. Kim. Phys. Rev. E, 74:045101(R), 2006.

35. L. de Arcangelis, C. Perrone-Capano, and H. J. Herrmann. Phys. Rev. Lett., 96:028107, 2006.

36. J. Hesse and T. Gross. Front. Syst. Neurosci., 8:166, 2014.

37. S. Ciliberto and P. Bigazzi. Phys. Rev. Lett., 60(4):286, 1988.

38. U. Frisch. Turbulence: The legacy of Kolmogorov. Cambridge University Press, 1995.

39. B. Hille. Ion channels of excitable membranes, 3d edition. Sinauer associates, Inc., 2001.

40. U. Parlitz and W. Lauterborn. Phys. Rev. A, (36):1428, 1987.

41. A. Panfilov and P. Hogeweg. Phys. Lett. A, 176:295, 1993.

42. L. Landau and E. M. Lifchitz. Physique statistique. édition MIR Ellipses, 4th edition, 1994.

43. Y. J. Chen, S. A. Chen, M. S. Chang, and C. I. Lin. Cardiovascular Res., 48:265, 2000 .

44. G. Bub, A. Shrier, and L. Glass. Phys. Rev. Lett., 88(5):058101, 2002.

45. G. Bub, A. Shrier, and L. Glass. Phys. Rev. Lett., 94:028105, 2005.

46. H.-Z. Wang, J. L. Jian, F. L. Lemanski, and R. D. Veenstra. Biophys. J., 63:39, 1992.

47. J. Neyton and A. Trautmann. J. Exp. Biol., 124:93, 1986.

48. M. C. Cross and P. C. Hohenberg. Rev. Mod. Phys., 65(3):851, 1993.

49. P. Glansdorff and I. Prigogine. Thermodynamic Theory of structure, stability and fluctuations. Wiley-Interscience, John Wiley \& Sons Ltd., 1971.

50. I. Prigogine and G. Nicolis. Quarterly Reviews of Biophysics, 4(2 \& 3):107, 1971.

51. P. Bergé and M. Dubois. Le journal de physique-Lettres, (10).

52. E. G. Daoud et. al. Circulation, 94:1600, 1996.

53. H. Fukuyama and P. A. Lee. Phys. Rev. B, 17(2):535, 1978.

54. P. A. Lee and T. M. Rice. Phys. Rev. B, 19(8):3970, 1979.

55. M. J. Rice. Phys. Rev. Lett., 36(8):432, 1976.

56. G. Grüner. Rev. Mod. Phys., 60(4):3970, 1979.

57. P. C. Hohenberg and P. C. Hohenberg. Rev. Mod. Phys., 49(3):435, 1977.

58. C. R. Myers and J. P. Sethna. Phys. Rev. B, 47(17):11171, 1993.

59. D. S. Fisher. Phys. Rev. Lett., 50(19):1486, 1983.

60. H. Hinrichsen. Advances in Phys., 49(7), 2000. 